MSO3-02

\section{Structural and mechanistic insights into DNA transposition facilitate genetic tool development}

Orsolya Barabas $^{1}$, Irma Querques ${ }^{1}$, Cecilia Zuliani ${ }^{1}$, Franka Voigt ${ }^{1}$, Lisa Wiedemann², Csaba Miskey², Zoltán Ivics²

1. Structural and Computational Biology Unit, European Molecular Biology Laboratory (EMBL), Heidelberg, Germany

2. Division of Medical Biotechnology, Paul Ehrlich Institute, Langen, Germany

email: barabas@embl.de

The DNA transposon Sleeping Beauty $(S B)$ is an efficient gene delivery vector with broad utilities in vertebrate genetics and human gene therapy. However, the molecular principles of its transposition remain elusive, greatly hampering rational design of tailored $S B$ variants. In this talk, I will present integrative high-resolution insights into $S B$ transposition and demonstrate how these enable directed engineering of advanced transposon tools. Using a combination of crystallography, SAXS, biochemical, biophysical techniques, molecular modelling and fluorescence cell imaging, we have dissected the assembly pathway of $S B$ 's active transposition machinery and showed that it follows a distinct pathway from other known transposons. We visualized the structures of nucleoprotein complexes involved in the onset of transposition, elucidating the basis of the differences between $S B$ and a canonical human element at high resolution. This showed that $S B$ 's unique assembly mode critically underlies its exceptional efficiency and allowed us to develop a strategy to chemically control transposition rates in living cells. Guided by our structure of the SB transposase catalytic domain, we further developed custom-made $S B$ variants with increased activity and safety, clearly illustrating the power of structure-guided rational protein design for the advancement of molecular tools.

Keywords: protein-DNA complex, integrating structure and function, genetic engineering
MSO3-03

\section{Observations of ultra-fast light-induced processes in proteins using time-resolved serial femtosecond crystallography}

\author{
Gabriela Nass Kovacs ${ }^{1}$ \\ 1. Biomolecular Mechanisms, MPI for medical Research, \\ Heidelberg, Germany \\ email: gabriela.kovacs@mpimf-heidelberg.mpg.de
}

The ultrashort and highly intense pulses of new X-ray sources, the X-ray Free-electron lasers (XFELs), open the sub-ps time domain for time-resolved crystallography using small crystals that can be efficiently photolyzed. This is particularly appealing for the study of photosensitive proteins, which contain a light-absorbing chromophore that allows exploitation of light energy as a resource or as a carrier of information initiating intra- or intercellular signaling. The ultra-fast light-induced events comprising double-bond isomerization have been the subject of intense research for decades and have been spectroscopically well characterized. However, direct structural information on the excited state and intermediate structures necessary to understand the underlying mechanisms has been inaccessible until recently. We present recent insight on the initial events in photoisomerization obtained by time-resolved serial femtosecond crystallography experiments in combination with time-resolved spectroscopy and quantum chemical calculations. This comprehensive mechanistic insight is not only important for the fundamental understanding of light-driven processes but has practical impact on future developments of fluorescent proteins for optical nanoscopy or retinal proteins for optogenetics.

Keywords: XFELs, time-resolved crystallography 